COMECHINGONIA. REVISTA DE ARQUEOLOGÍA

Número 14, 2011, pp. 97-113, Córdoba

ISSN 0326-7911

\title{
RECURSOS LÍTICOS, APROVISIONAMIENTO Y ASPECTOS TEMPORALES DE FUENTES DE ABASTECIMIENTO EN AMAICHA DEL VALLE, TUCUMÁN, ARGENTINA

\author{
Carolina Somonte ${ }^{1}$ y Carlos Baied ${ }^{2}$
}

\begin{abstract}
${ }^{1}$ Instituto Superior de Estudios Sociales (ISES) - CONICET e Instituto de Arqueología y Museo, Facultad de Ciencias Naturales e Instituto Miguel Lillo, Universidad Nacional de Tucumán. San Martín 1545, T4000CWE Tucumán, carosomonte@hotmail.com

2Instituto de Arqueología y Museo, Facultad de Ciencias Naturales e Instituto Miguel Lillo, Universidad Nacional de Tucumán. San Martín 1545, T4000CWE Tucumán, cbaied@yahoo.com
\end{abstract}

Presentado el: 2/12/2010 - Aceptado 23/03/2011

\section{Resumen}

Este trabajo aborda la problemática del aprovisionamiento de recursos líticos y aspectos cronológicos de las fuentes de abastecimiento a partir del espacio donde se encuentra emplazado el sitio arqueológico Planchada La Puntilla (Amaicha del Valle, Tucumán, Argentina). Se presentan los atributos generales de la base regional de recursos líticos, las materias primas utilizadas y sus fuentes de aprovisionamiento. Específicamente se discute la utilidad del concepto de fuente terciaria en relación al uso/explotación de las fuentes de aprovisionamiento en el largo plazo.

Palabras claves: Aprovisionamiento, Fuentes Terciarias, Recursos Líticos, Andesita.

\section{Abstract}

This paper addresses the procurement of lithic raw materials and chronological aspects of procurement sources in the broad area within which the archaeological site Planchada La Puntilla (Amaicha del Valle, Tucumán, Argentina) is located. We start with an overview of the general attributes of the regional lithic resource base, followed by a characterization of the lithic raw materials used and, finally by a discussion of procurement quarries. More specifically, we discuss the usefulness of the tertiary source concept in relation to long-term use/exploitation of procurement sources.

Key Words: Lithic Procurement, Tertiary Sources, Lithic Resources, Andesite.

\section{Introducción}

El término fuente terciaria fue definido por Church (1995:19) como "a lithic artifact assemblage that forms a source of lithic material for later populations". Este concepto permite hacer frente a 
situaciones particulares en las que tiene lugar el aprovisionamiento de recursos líticos. Se refiere a escenarios de fuentes de abastecimiento que son producto de procesos antrópicos, a diferencia de los tipos de fuentes de origen natural como son los casos de las fuentes primaria y secundaria (Church 1995; Luedtke 1979; Nami 1992, entre otros).

En este trabajo se entiende que las fuentes terciarias están conformadas por importantes cantidades de materiales líticos que incluyen distintas etapas de la secuencia de producción y que por alguna razón han sido descartados, quedando durante cientos o miles de años como un agregado a disposición de potenciales futuros usuarios. El término está relacionado con el aspecto temporal del uso de las fuentes, tema que, en general, ha recibido poca atención por parte de investigadores. Esto se debe, en parte, a la naturaleza general de toda fuente de aprovisionamiento, cuyas características impiden, a prima facie, responder preguntas tales como ¿Desde cuándo se explota una fuente de aprovisionamiento? ¿Cómo se evalúa la intensidad y/o recurrencia en el uso de la misma? ¿Cómo se verifica el cambio de estrategias de aprovisionamiento implementadas en su explotación?

Las respuestas a algunas de estas preguntas requieren que las fuentes de aprovisionamiento cuenten con un indicador temporal que de cuenta de su utilización en el largo plazo. Para el área que nos ocupa, un indicador temporal a tener en cuenta es el barniz de las rocas, una película muy delgada constituida por aproximadamente dos tercios de minerales arcillosos cementados a una roca soporte $y$, típicamente, por un quinto de manganeso y oxido - hidróxido de hierro (Dorn 2007). Esta película afecta los rodados naturales, artefactos líticos y estructuras dispuestas sobre diversas geoformas del área de estudio.

Teniendo en cuenta los procesos y tiempos requeridos para la formación y desarrollo del barniz de las rocas, se considera a este fenómeno como un indicador geo-cronológico en lo que hace a la arqueología de Amaicha del Valle (Somonte y Collantes 2007, Somonte 2009). Si bien esta pátina no es la única que afecta a los artefactos líticos recuperados en el área, la ventaja que tiene sobre las restantes alteraciones es que ésta puede ser datada mediante un análisis conocido como varnish microlamination (en adelante VML) (Dorn 2007; Liu 2003; Liu y Broecker 2007, 2008; Liu y Dorn 1996). Planchada La Puntilla es uno de los sitios arqueológicos de la localidad, que posee un registro lítico afectado por el barniz de las rocas y que se encuentra emplazado en una fuente secundaria en la que se cuenta, a su vez, con algunos sectores que aqui definimos como fuente terciaria (sobre este tema volveremos en detalle mas adelante en este trabajo). Esta situación permite profundizar la discusión entre aprovisionamiento de recursos líticos y la historia ocupacional de otros sitios del área en el marco de diversos procesos de reclamación artefactual (Schiffer 1987). Desde una perspectiva tafonómica, estos temas cuentan con antecedentes importantes (Borrazzo 2006; Hiscock 2007, entre otros).

El objetivo de este trabajo es, entonces, dar a conocer las fuentes de aprovisionamiento de materias primas y revalorizar el estudio de los aspectos temporales en el uso de esas fuentes, tomando como caso de estudio el sitio Planchada La Puntilla. En este sentido se evalúa la utilidad del concepto de fuente terciaria como herramienta válida para dar cuenta de variabilidad temporal en los escenarios asociados al aprovisionamiento. 


\section{Entorno ambiental y el sitio arqueológico}

Amaicha del Valle es un valle tectónico que se encuentra a 2000 msnm en el Departamento de Tafí del Valle, Provincia de Tucumán, Argentina. Pertenece al ambiente morfoestructural de las Sierras Pampeanas y está limitado por los cordones montañosos de las Sierras del Aconquija y Cumbres Calchaquíes. Este valle tectónico se inicia en el Abra del Infiernillo con una extensión de $16 \mathrm{~km}$ y se dirige hacia el NO hasta unirse con el valle de Santa María (Bossi et al. 1984) (Figura 1).

El sitio Planchada La Puntilla está localizado en la localidad de La Puntilla, cerca de la villa de Amaicha del Valle, entre los ríos Las Salinas y Amaicha. Se encuentra a 1990 msnm

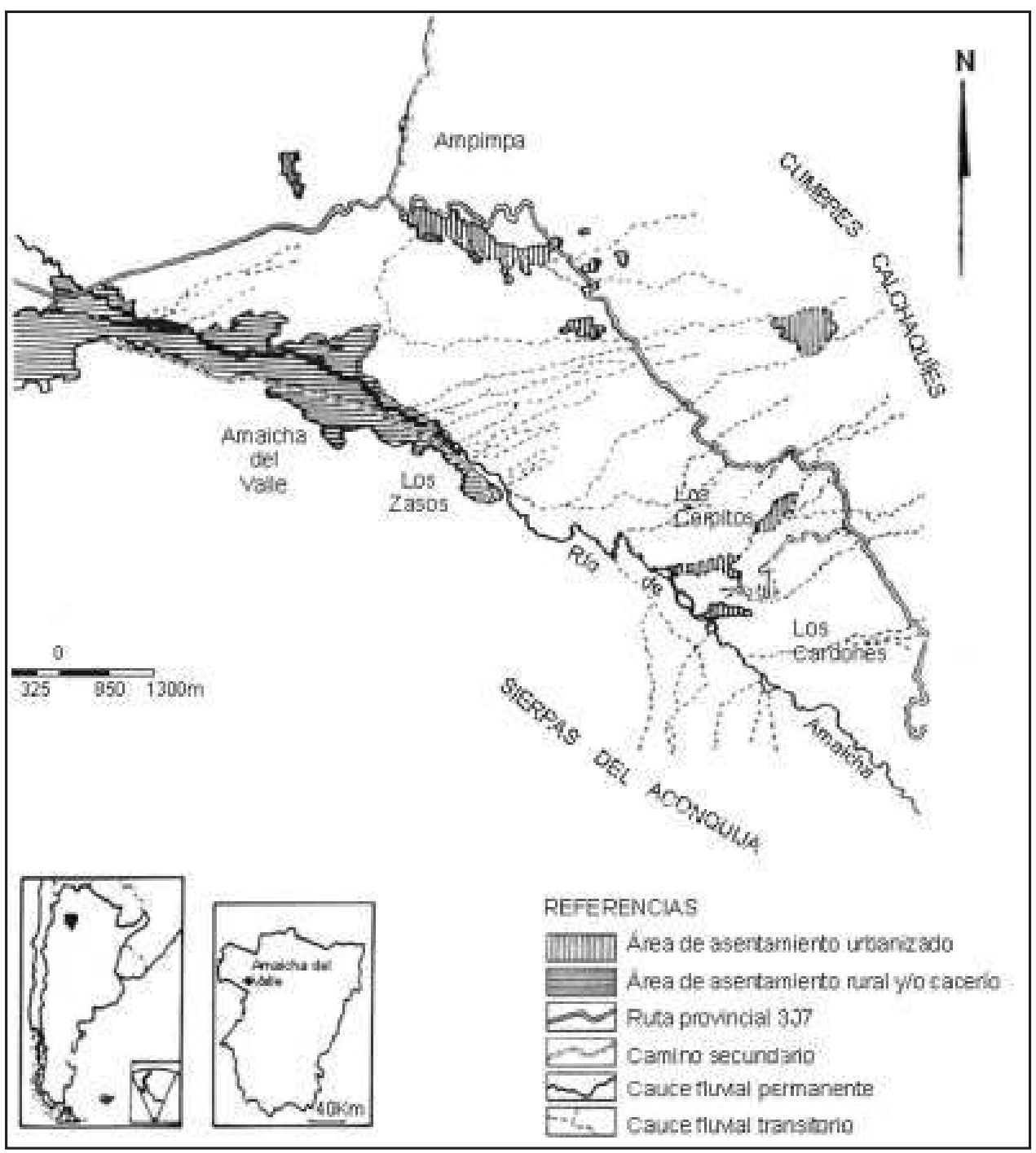

Figura 1. Mapa general de Amaicha del Valle 
a los $26^{\circ} 35^{\prime} 49^{\prime \prime}$ lat. Sur y $65^{\circ} 56^{\prime} 97^{\prime \prime}$ long. Oeste sobre una superficie de glacis que se extiende aproximadamente $200 \mathrm{~m}$ en sentido N-S y $1000 \mathrm{~m}$ en sentido E-O. Este sitio puede dividirse en dos grandes sectores. Uno de ellos se caracteriza por la presencia de áreas a cielo abierto, separadas unas de otras, que cuentan con evidencia de explotación in situ de los recursos líticos disponibles en el glacis. Allí se concentran algunos conjuntos líticos en superficie afectados por barniz de las rocas, cuyas dataciones mediante la técnica VML indican la ocupación del sitio desde momentos tempranos. Ha sido posible, además, registrar información sobre la reocupación del mismo hasta el período Tardío, en base a las características arquitectónicas y constructivas identificadas en algunos de los recintos, a lo que se suma la presencia de fragmentos cerámicos del estilo Santamariano (Somonte 2009).

El segundo sector está relacionado con la presencia de seis recintos habitacionales y una estructura en forma de arco simple (Figura 2). En general, estas estructuras presentan una mala conservación, dado que hay casos de desmantelamientos parciales (Recintos 1 y 3), en otros casos totales (Recintos 5 y 6), a lo que se suma evidencias de huaqueo (Recinto 7). Los recintos, confeccionados con rocas sin cantear, son simples y poseen dimensiones similares de aproximadamente $5 \mathrm{~m}$. Las diferencias constructivas observadas en estos hacen que no sea posible describir un diseño arquitectónico homogéneo para el conjunto de estructuras del sitio Planchada La Puntilla ya que no guardan relación constructiva estricta, más allá de su emplazamiento espacial de relativa proximidad.

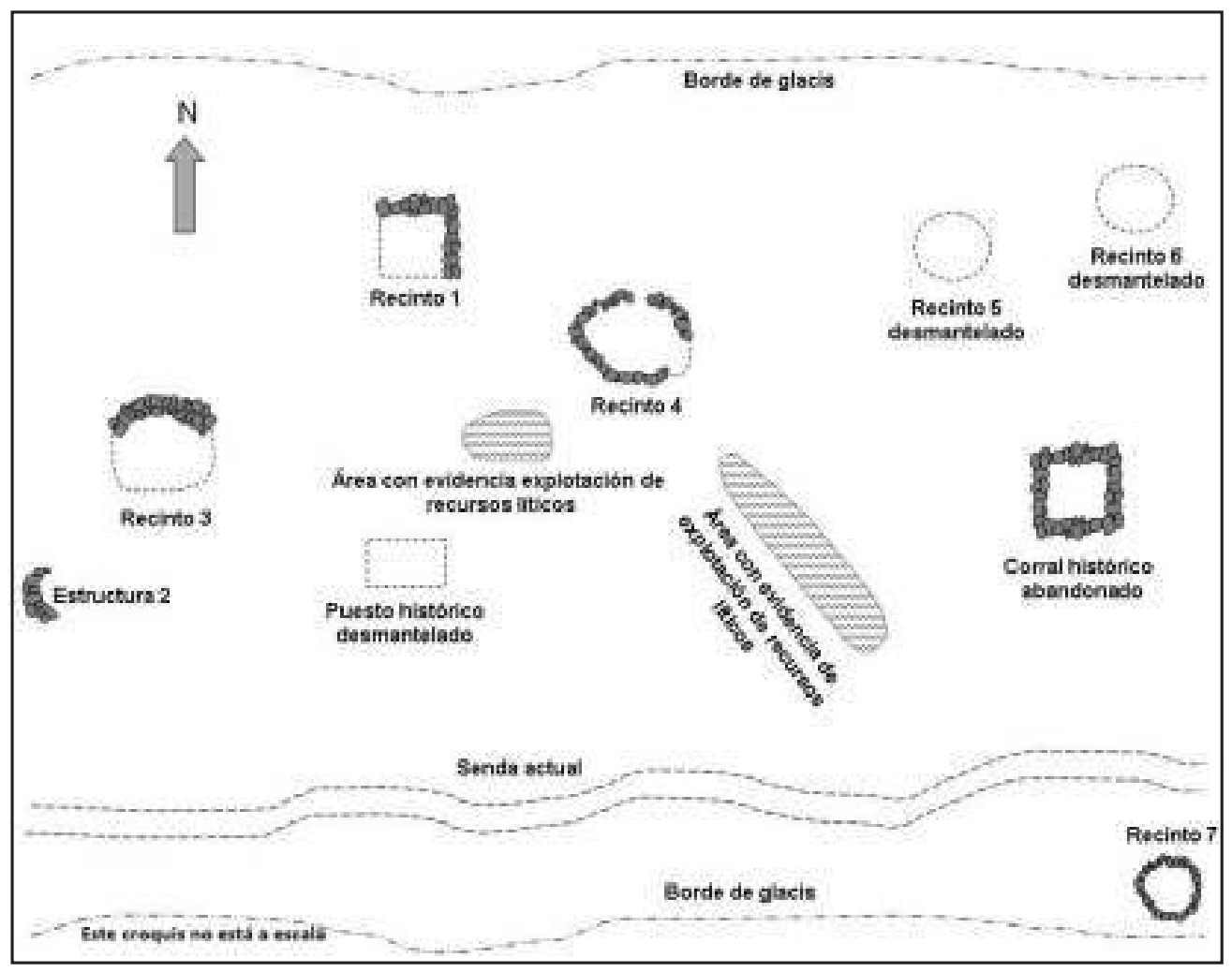

Figura 2. Croquis del sitio Planchada La Puntilla 
El recinto denominado $\mathrm{R} 4$ presenta un mayor cuidado con respecto a los otros, en lo que hace a la construcción de los cimientos y otros elementos arquitectónicos, como ser la presencia de una rampa de acceso y un piso cubierto con una lechada de arcilla (Somonte 2009). De este recinto se recuperaron solamente núcleos de dimensiones importantes y desechos de talla producto de la regularización de filos de artefactos que no están en el sitio. Estos hallazgos (este recinto fue el único en el que se recuperó material arqueológico en subsuperficie), las características generales de los recintos y la situación contextual nula en gran parte de los mismos, en conjunto indicarían que probablemente se trate de un sitio de actividades específicas, donde aparentemente no hubo necesidad de una permanencia prolongada, dado que no hay evidencias que permitan afirmar tal situación. Estos dos sectores remiten a diferentes momentos temporales en el uso/explotación de ese espacio.

\section{Disponibilidad de recursos líticos: Hacia la definición de la base regional en Amaicha del Valle}

La base regional de recursos líticos del área estudiada indica que, entre los recursos que ofrecen cierta calidad para la talla (sílices, cuarcitas y andesitas), los que se encuentran mayormente disponibles son las variedades de andesitas en una relación de nueve a uno para con otros potenciales recursos. Por su parte, los recursos como las cuarcitas y sílices (xilópalo o madera silicificada) poseen una distribución acotada espacialmente. Asimismo, son abundantes las rocas metamórficas, aunque sus propiedades o cualidades no las hacen adecuadas para las actividades de producción de artefactos tallados. Independientemente de la proporción de estos recursos, es importante destacar que todos los tipos rocosos presentan tamaños variables, pero aptos para su explotación (Somonte 2009).

Por otro lado, existen ciertas unidades sedimentarias que se formaron por aporte de material volcánico proveniente del Complejo Portezuelo de las Ánimas¹ (González 1990; González et al. 2000; González y Barreñada 1993). Estas formaciones del Terciario Superior forman parte del Grupo Santa María, y afloran en todo el Valle de Santa María ${ }^{2}$. Las distintas unidades litológicas se manifiestan como paralelas al río Santa María, sobre la margen derecha y también en la depresión tectónica del río Amaicha y sus alrededores (Bossi et al. 1984; Gavriloff et al. 1998; González et al. 2000, 1998). Esto indicaría que los materiales volcánicos (de composición andesítica su mayoría) del Complejo tendrían amplia distribución en el área de estudio (Tabla 1).

\section{Metodología de registro en las fuentes de aprovisionamiento}

El establecimiento de las potenciales canteras se realizó en función de: (1) la información de la base regional de recursos líticos, (2) la localización de diversos sitios arqueológicos (aunque en este trabajo se presentan los resultados de uno en particular), (3) las consultas con geólogos y geomorfólogos familiarizados con el área y los tipos rocosos (4) las tareas de campo llevadas a cabo en lugares donde podrían estar presentes los afloramientos de las formaciones sedimentarias. Las prospecciones se realizaron en diversos lugares y apuntaron a evaluar -sobre todo en aquellas zonas donde se registró evidencia de explotación de fuentesla variabilidad de recursos líticos ofrecidos por las fuentes en cuanto a su ubicación y acceso desde el sitio; distribución, concentración y forma en que se presentan los recursos en estado natural (dimensiones, cantidad, etc.). Esto, junto a la información del sitio arqueológico permitió discutir qué rocas aparecen, su diversidad, posible origen y modo de explotación. 


\begin{tabular}{|c|c|c|c|}
\hline RECUASOLIIICO & DISPONIBLE EN & APARECE EN FORMA DE & TAMAREO PROMEDIO \\
\hline Vulcanitas (andesitas) & $\begin{array}{l}\text { Compiejo Portezuela de Las Animas } \\
\text { Formación Saladilo } \\
\text { Formacion San José } \\
\text { Formacion Chiquimil } \\
\text { Formación Andalhuals } \\
\text { Formación Los Corralas } \\
\text { Formación Yasyamayo }\end{array}$ & $\begin{array}{l}\text { Brechas (piroclasticas y } \\
\text { lopiliticas) } \\
\text { Conglamerados } \\
\text { Lentes }\end{array}$ & Entre $5.80 \mathrm{~cm}$ \\
\hline $\begin{array}{l}\text { Silices } \\
\text { (madera silicificada } \\
\text { y otras tipes) }\end{array}$ & $\begin{array}{l}\text { Formación San José } \\
\text { Formación Chiquimil } \\
\text { Formación Andaihuala }\end{array}$ & $\begin{array}{l}\text { Trozes de tronco } \\
\text { silicificado }\end{array}$ & Entre $5.60 \mathrm{~cm}$ \\
\hline Cuareitas & Basamenfo Sierras del Aconquija & Descanosida & \\
\hline Quarzo & $\begin{array}{l}\text { Formacién San Josd } \\
\text { Formación Las Arcas } \\
\text { Formacién Andalhuald } \\
\text { Formecion Yasyamayo }\end{array}$ & $\begin{array}{l}\text { Pegmatias } \\
\text { Conglomerados } \\
\text { Inyecciones laminarss } \\
\text { yo en capas }\end{array}$ & Entre $5.30 \mathrm{~cm}$ \\
\hline Metamóficas & $\begin{array}{l}\text { Formacion San Jogé } \\
\text { Formación Las Atcas } \\
\text { Formacion Chiquimil } \\
\text { Formación Andalhuald } \\
\text { Formecion Los Corrales } \\
\text { Formación Yasyamayo. }\end{array}$ & $\begin{array}{l}\text { Conglamerados } \\
\text { Lentes }\end{array}$ & Entre $5.80 \mathrm{~cm}$ \\
\hline
\end{tabular}

Tabla 1. Síntesis base regional de recursos liticos de Amaicha del Valle.

En el caso particular del glacis donde se encuentra emplazado el sitio Planchada La Puntilla, el relevamiento y muestreo se realizó a través del trazado de dos transectas paralelas de $50 \mathrm{~m}$ de longitud y $2 \mathrm{~m}$ de ancho cada una. Cada una de estas unidades fue dividida, a su vez, cada $2 \mathrm{~m}$ de manera de obtener un reticulado del área de muestreo. En cada subunidad se completó una ficha en la que se consignaron variables como tipo de roca, abundancia, dimensiones, morfología de los clastos, forma en que se encuentra disponible la roca, alteración superficial, posición de la pieza al momento de la recolección, color, tamaño, calidad, entre otras. Esto se repitió a lo largo de toda la transecta.

\section{Materias primas líticas: Su identificación}

En los conjuntos líticos analizados del sitio Planchada La Puntilla se identificó una variada gama de materias primas las que fueron determinadas macroscópicamente. Los criterios establecidos para esta determinación estuvieron relacionados tanto con las propiedades físico-químicas como con otras propiedades cualitativas. Estos criterios fueron: tipo de roca, color de la matriz, tamaño relativo de los cristales de la matriz, composición de los fenocristales, forma, tamaño y densidad relativa de los fenocristales y fractura.

Entre las materias primas se encuentran, en orden de importancia por su frecuencia de aparición: andesitas en distintas variedades, metamorfitas -esquistos y gneises-, cuarzo en sus variedades cristalino y lechoso, cuarcitas y sílices (xilópalo y otros). Teniendo en cuenta 
que la mayor proporción de recursos utilizados como materias primas líticas corresponde a las andesitas, se profundizó en la identificación macroscópica de las mismas. Esta identificación se reforzó mediante la comparación entre muestras arqueológicas y muestras de mano geológicas del Complejo Volcánico Portezuelo de Las Ánimas ya que sobre estas últimas se hicieron cortes petrográficos (González 1990). Esta comparación permitió establecer preliminarmente una relación entre estos recursos y las materias primas líticas registradas en los conjuntos arqueológicos. Los cortes delgados realizados sobre las muestras geológicas indican la existencia de, al menos, tres grupos de rocas volcánicas: (1) aquellas de composición fenoandesítica de tonalidades gris oscura, clara y pardo rojizas; (2) las fenoandesitas con tendencia a basaltos de color gris oscuro que poseen pasta muy fina y fractura concoidea $y$, finalmente, (3) las fenoandesitas de tonalidades grises fundamentalmente.

Las variedades de rocas volcánicas identificadas en los conjuntos arqueológicos fueron reunidas dentro de tres grandes grupos:

I - Andesita variedad B o Basandesita: Dentro de este grupo están incluidas aquellas andesitas básicas o basandesitas - con tendencia a basaltos - comunes en la parte superior de la sección tipo del Complejo Portezuelo de las Ánimas, Sierras del Aconquija (González 1990). Este grupo incluye rocas que presentan un color gris oscuro, la matriz es afanítica y no presenta fenocristales, lo que estaría inhibiendo a la roca de la existencia de zonas de debilidad para la talla. En general, este conjunto incluye rocas que pertenecen al segundo de los grupos surgidos de los cortes petrográficos. La fractura que presentan es concoidea y por sus atributos es la que mejor calidad ofrece para la talla.

II - Andesita variedad G: Dentro de este grupo están incluidas aquellas fenoandesitas, también comunes en la sección tipo del Complejo Portezuelo de las Ánimas y en el río Amaicha. Se corresponde con el tercero de los grupos definidos por cortes petrográficos. La característica que la diferencia de la variedad $B$ es la presencia de fenocristales de minerales leucocráticos y melanocráticos (ninguno supera los $5 \mathrm{~mm}$ ) que se presentan en diversas formas, redondeada y acicular. La fractura de esta variedad es concoidea, pero la presencia de fenocristales genera fracturas irregulares durante las tareas de talla.

III - Andesita variedad P: Dentro de este grupo están incluidas las rocas de composición fenoandesítica comunes en la parte inferior de la secuencia del Complejo Portezuelo de las Ánimas, presentes también entre los materiales del río Amaicha. Son macizas, de color pardo rojizo y textura porfírica constituida por minerales melanocráticos, en sus diversas formas, y también leucocráticos de forma redondeada. Está asociada al primero de los grupos de andesitas surgidos a partir de los cortes petrográficos. La fractura de esta variedad es concoidea, pero la presencia de fenocristales, al igual que en la variedad G, provoca fracturas irregulares durante las tareas de talla.

Por otro lado, los criterios de identificación de recursos como cuarcitas, sílices y cuarzo, estuvieron relacionados especialmente con el color de la matriz, ya que las restantes características no fueron relevantes para la diferenciación de las variedades de estos tipos de roca. De esta manera, las cuarcitas, todas de grano mediano a fino, fueron diferenciadas por los colores que presentan, variando su tonalidad dentro de la gama de los blancos, rosas y verdes. Los sílices se diferenciaron en variedades de colores rosados y marrones. Particularmente, estos últimos corresponden a xilópalo. Finalmente, el cuarzo se encuentra disponible en su variante cristalina y blanca. 


\section{Fuentes de aprovisionamiento: Su localización y evidencias de explotación}

En esta parte del trabajo se busca responder a preguntas tales como ¿Dónde se encuentran disponibles los recursos líticos seleccionados por las sociedades prehispánicas para la producción lítica? ¿Qué tipo de fuentes de aprovisionamiento son? ¿Cuál es la evidencia lítica de explotación de estas fuentes?

Los recursos líticos aparecen distribuidos en distintos sectores del valle tectónico de Amaicha del Valle. Las fuentes de aprovisionamiento de materias primas líticas son fundamentalmente secundarias y terciarias, ya que no existen afloramientos in situ de rocas, y sólo el caso de la cantera de xilópalo puede ser considerado como fuente primaria de aprovisionamiento.

En primer lugar, las variedades de andesitas se encuentran disponibles en fuentes secundarias y terciarias (sensu Church 1995). Con respecto a las fuentes secundarias, una de ellas es el río Amaicha, afluente del río Los Corrales (ambos relacionados a la formación Los Corrales y al Complejo Volcánico Portezuelo de las Ánimas). Esta fuente está ubicada a menos de $1 \mathrm{~km}$ de distancia del sitio Planchada La Puntilla. En el cauce de este río abunda fundamentalmente la variedad de andesita G, y en menor proporción las variedades B y P, todas disponibles en forma de nódulos rodados, distribuidos de manera concentrada (la variedad G) y más bien dispersa (las variedades B y P) a lo largo del cauce. Se constató su explotación a la altura de la localidad de Los Cardones, donde se encuentra el sitio Bajo Los Cardones, además de otros sitios arqueológicos cronológicamente más tempranos (Campo Blanco) y más tardíos (Los Cardones).

La evidencia lítica de explotación de esta cantera está dada por la presencia de numerosos núcleos semi-enterrados en el lecho del cauce, los cuales a juzgar por sus dimensiones fueron, originalmente, nódulos superiores al metro de diámetro. Algunos de estos núcleos, poseen numerosas extracciones, cuyas características permiten sostener que han sido producidas mediante tareas de talla y no pueden ser atribuidas a efectos del rodamiento por acción hídrica. Pero además, a medida que se accede a las terrazas fluviales, pueden observarse pequeños sectores que no superan los $2 \mathrm{~m}^{2}$, con evidencia de eventos de talla aislados núcleos y lascas fundamentalmente- que podrían ser considerados como pequeñas áreastaller presentes en lugares puntuales cercanos al cauce. En conjunto, esta evidencia indicaría la realización de tareas de reducción primaria. Por otro lado, en el río Amaicha a la altura del sitio Planchada La Puntilla, también se han realizado prospecciones sistemáticas tendientes a la búsqueda de evidencia lítica de la explotación de las variedades de andesitas en esta fuente, las que no arrojaron resultados.

Otra de las fuentes secundarias consideradas en este trabajo es el mismo río Las Salinas, afluente del río Amaicha. Esta fuente potencial está a $300 \mathrm{~m}$ del sitio Planchada La Puntilla. Allí se encuentran rodados de las variedades de andesitas B, G y P, los cuales se presentan de la misma forma que en el río Amaicha, aunque disponibles en proporciones bastante menos importantes. Esto significa que el material se presenta en forma de nódulos rodados distribuidos de manera dispersa a lo largo del cauce. En esta fuente de aprovisionamiento no se ha constatado evidencia lítica de su explotación, por lo que se mantiene su carácter potencial. 
Otro tipo de fuentes secundarias donde se encuentran disponibles las variedades de andesitas G, B y $P$, son los depósitos de materiales rocosos dispuestos sobre diferentes superficies de glacis y abanicos aluviales cuya particularidad es que están afectados por barniz de las rocas. Se debe recordar que el sitio Planchada La Puntilla, entre otros, se encuentra asociado a la fuente misma, teniendo de esta forma los recursos líticos 'a la mano', es decir inmediatamente disponibles en el sentido dado por Civalero y Franco (2003). En estas fuentes, las andesitas se presentan en forma de nódulos redondeados y, en algunos casos, más bien aplanados, dando el aspecto de 'nódulos tabulares', sin llegar a serlo en un sentido estricto. Estos nódulos están ampliamente disponibles y distribuidos de forma concentrada sobre la superficie de las geoformas.

Teniendo en cuenta las características de estos depósitos, estas fuentes de andesitas pueden ser consideradas secundarias en el sentido otorgado por Luedtke (1979) y Church (1995) ya que los materiales rocosos dispuestos sobre estas superficies son producto del transporte desde sus fuentes primarias o de origen. Sin embargo, las andesitas también están disponibles en este tipo de fuentes bajo la forma de materiales líticos tallados que se encuentran en distintas etapas de producción y distribuidos en forma concentrada en determinados sectores de estas superficies. La presencia de abundantes núcleos, lascas corticales e internas diversas, artefactos bifaciales y unifaciales (con distintos grados de formatización y estados de fragmentación), en conjunto evidenciaría, en primera instancia, la explotación de estas canteras. Sobre este aspecto volveremos más adelante en el trabajo. Las prospecciones en el área involucraron algunas otras fuentes similares en sus características y asociadas a sitios arqueológicos, las que se denominaron Campo Grande y La Loma. Ambas se encuentran a una distancia aproximada de $2 \mathrm{~km}$ del sitio Planchada La Puntilla (Figura 3).

En segundo lugar, el xilópalo se encuentra disponible en una fuente primaria de aprovisionamiento, localizada en Tio Punco a $10 \mathrm{~km}$ al norte del sitio Planchada La Puntilla. Esta fuente consiste en una zona sobreelevada de forma ovalada compuesta en su totalidad por este material fósil, cuya altura no alcanza los $100 \mathrm{~m}$ y que cubre una superficie de aproximadamente $100 \mathrm{~m}$ de largo por $60 \mathrm{de}$ ancho. Las prospecciones en la fuente permiten sostener que este recurso se encuentra disponible bajo la forma de trozos de tronco silicificado, distribuidos de manera concentrada en una superficie reducida en comparación con la disposición areal que presentan otros recursos. La materia prima en esta fuente se presenta dentro de las tonalidades marrones y no hemos registrado material silíceo de otro color. En este sentido, queda sin haber sido localizada la fuente de sílices rosados.

La evidencia lítica de explotación de esta fuente es abundante en extremo y prácticamente no existen nódulos, es decir material sin explotar. El motivo de esta sobre-representación se debe a los usos contemporáneos recientes que obliteraron totalmente la posibilidad de recuperar alguna evidencia de explotación prehispánica. Esto hace que se deba considerar a esta fuente como potencial. La base regional de recursos líticos indica la existencia de otras localidades de estas características hacia el sector de Los Colorados, al SO de la villa de Amaicha del Valle. Sin embargo, las prospecciones realizadas en esta zona no condujeron al hallazgo de dichos afloramientos. 


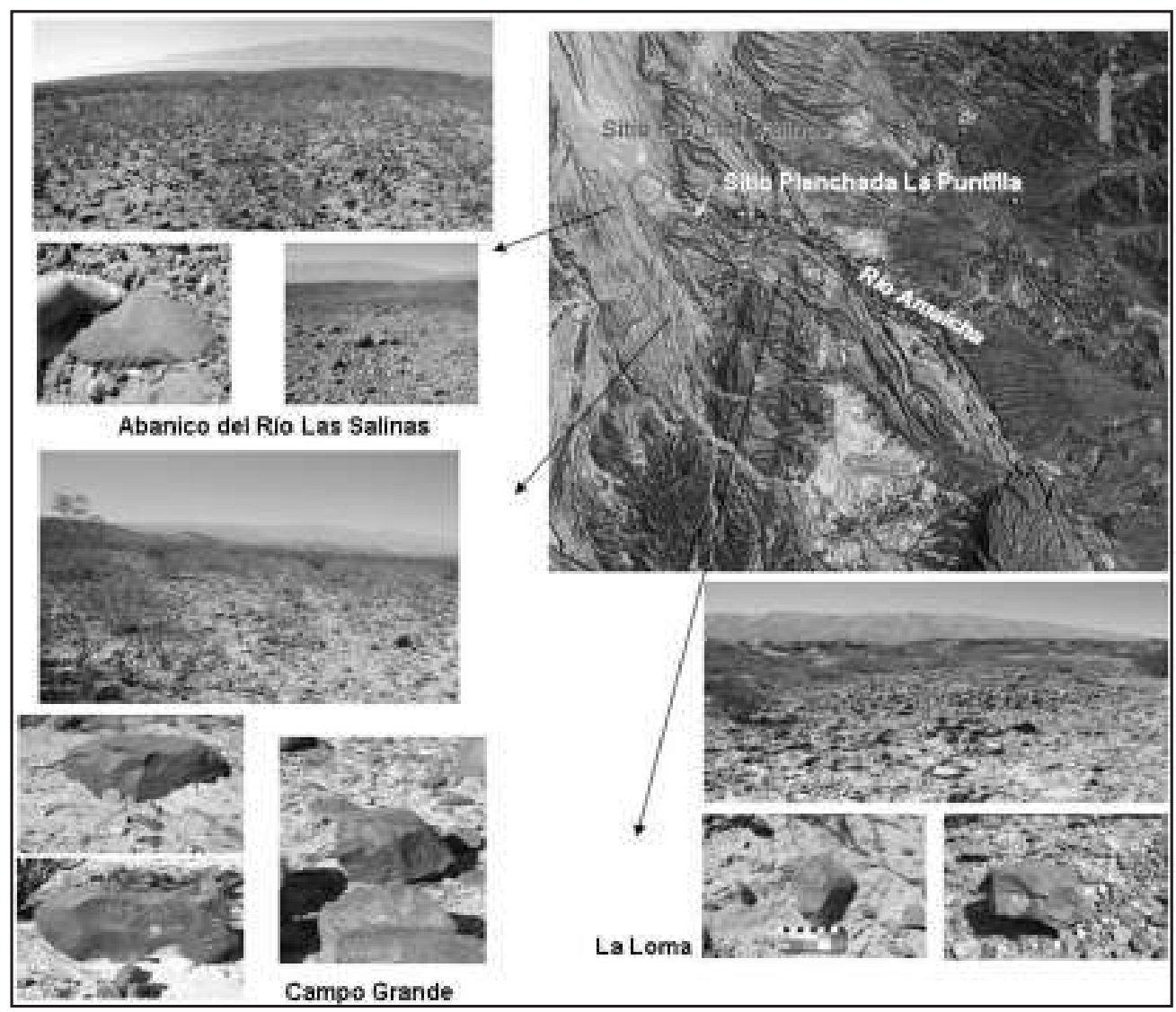

Figura 3. Fuentes de andesitas en La Puntilla y Río Las Salinas.

En tercer lugar, la cantera principal de cuarzo y metamorfitas es la fuente secundaria del río Amaicha. Su disponibilidad, a diferencia de las vulcanitas, es mucho menor en proporción, pero similar en lo que hace a la forma en que se presenta este material. Sin embargo, no se ha registrado evidencia de explotación de estos recursos en la fuente mencionada, siendo de este modo una cantera potencial. A esta fuente secundaria se debe sumar, además, las superficies de glacis, donde se encuentran disponibles algunos nódulos de rocas metamórficas, aunque en menores proporciones que las andesitas. En esta fuente, los nódulos de rocas metamórficas poseen dimensiones algo menores a las constatadas entre los rodados presentes en el río Amaicha.

En cuarto lugar, la fuente de cuarcitas aún no fue localizada. Sólo se sabe que se encuentra en algún sector del basamento de las Sierras del Aconquija, a más de $20 \mathrm{~km}$ de distancia de los sitios arqueológicos en cuestión (González 2009 com. pers.).

Más allá de las particularidades de cada fuente, es importante reconocer que los recursos líticos del área tienen lugar en concentraciones localizadas, cuyas fuentes potenciales y efectivas, fueron, en principio, identificadas en el campo (Tabla 2). El uso de estos recursos involucró costos de búsqueda, aprovisionamiento y procesamiento, los cuales estuvieron 
relacionados con la distribución y disponibilidad, y no con la accesibilidad, a las fuentes de estas materias primas.

\begin{tabular}{|c|c|c|c|c|c|}
\hline $\begin{array}{c}\text { FuENTES DE } \\
\text { ABASTECIMENTO }\end{array}$ & $\begin{array}{l}\text { Materia } \\
\text { Prawa }\end{array}$ & $\begin{array}{l}\text { TIPODE } \\
\text { FUENTE }\end{array}$ & $\begin{array}{l}\text { DISTANCLIA } \\
\text { AL SMTIO }\end{array}$ & $\begin{array}{c}\text { FOPMA DE REFRESENTACION } \\
\text { Y DISTRBUCION }\end{array}$ & $\begin{array}{l}\text { EVDERICLA DE } \\
\text { EXPLOTACION }\end{array}$ \\
\hline Fío Ansaicha & $\begin{array}{c}\text { Vulcavitas } \\
\text { Metamorltas } \\
\text { Cuarzo }\end{array}$ & Secundsria & - de $1 \mathrm{ken}$ & $\begin{array}{l}\text { Claslos o rodados de } \\
\text { datnbucaki concenteada }\end{array}$ & si \\
\hline Ria Las Salnas & $\begin{array}{l}\text { Vubcantas } \\
\text { Metsenoritas }\end{array}$ & Secundra & - de $1 \mathrm{~km}$ & $\begin{array}{l}\text { Clastos o rodadas de } \\
\text { astribucice dispersa }\end{array}$ & NO \\
\hline $\begin{array}{l}\text { Garis Campo } \\
\text { Grande }\end{array}$ & $\begin{array}{l}\text { Vulcritas } \\
\text { Melamertlas }\end{array}$ & $\begin{array}{l}\text { Secundena - } \\
\text { Yerciars }\end{array}$ & Enthe $2.5 \mathrm{~km}$ & 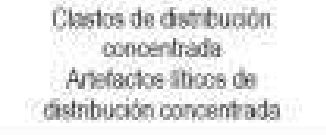 & si \\
\hline Glaxis La Loma & $\begin{array}{l}\text { Vulcanitas } \\
\text { Metameritas }\end{array}$ & $\begin{array}{l}\text { Secundana - } \\
\text { Terciaria }\end{array}$ & Enve $2.5 \mathrm{~km}$ & $\begin{array}{l}\text { Clasios de dstrtución } \\
\text { concenlrada } \\
\text { Arsetacios lificos do } \\
\text { distribucicin concentrada }\end{array}$ & $\mathrm{sr}$ \\
\hline $\begin{array}{l}\text { Abanwo Fio Las } \\
\text { Salnas }\end{array}$ & $\begin{array}{l}\text { Vuconitas } \\
\text { Metamoritas }\end{array}$ & $\begin{array}{l}\text { Secandris:- } \\
\text { Tercioni }\end{array}$ & - de $1 \mathrm{~km}$ & $\begin{array}{l}\text { Claslas de deatibucion } \\
\text { conoentrada } \\
\text { Artcfacbs iticos de } \\
\text { distritución concentrads }\end{array}$ & si \\
\hline $\begin{array}{l}\text { Glacis Planchada } \\
\text { La Puntila }\end{array}$ & $\begin{array}{l}\text { Vubcanitas } \\
\text { Metsenorltas }\end{array}$ & $\begin{array}{l}\text { Secoundona - } \\
\text { Tercisis }\end{array}$ & - de $1 \mathrm{~km}$ & 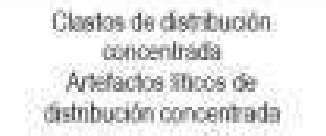 & si \\
\hline Tio Punco & $\begin{array}{l}\text { Madera } \\
\text { siicticada }\end{array}$ & Primatis & - de $20 \mathrm{~km}$. & $\begin{array}{l}\text { Trozos de tronoce de } \\
\text { distribución concentrada }\end{array}$ & s*t \\
\hline
\end{tabular}

Tabla 2. Síntesis de las fuentes de aprovisionamiento de andesitas de Amaicha del Valle.

Explotación a largo plazo de las fuentes de aprovisionamiento de andesitas: El rol del barniz de las rocas en el sitio Planchada La Puntilla

Los conjuntos líticos dispersos en superficie en el sitio Planchada La Puntilla -como ejemplo de una situación repetitiva en varios sitios de la zona- cuentan con artefactos que poseen negativos de lascados cubiertos por barniz, lo que significa una talla anterior a la depositación de esta pátina. Además, hay artefactos que poseen en algunas de sus caras y filos re-formatizaciones que afectan las áreas barnizadas, dejando expuesta la superficie fresca de la roca (o sea sin barniz). En algunos casos, inclusive, estas superficies 'frescas' poseen un desarrollo incipiente del barniz, adquiriendo una tonalidad menos intensa, que aquellas áreas más barnizadas. Esto significa que, siendo el barniz el proceso sedimentario más lento del mundo (Liu y Broecker 2000), estas sucesivas formatizaciones y reformatizaciones se produjeron con una interesante diferencia temporal que marca la presencia de algún tipo de proceso de reclamación. La reclamación en estas piezas está dada en base a la presencia diferencial en la intensidad del barniz en los negativos de lascados que hacen a las distintas formatizaciones de los filos. Entre los materiales que se presentan como ejemplos de las situaciones descriptas se destacan núcleos, artefactos formatizados y formas base que 
han sido analizados siguiendo los criterios propuestos por Aschero (1975 y 1987) y Aschero y Hocsman (2004) (Tabla 3 y Tabla 4).

\begin{tabular}{|c|c|c|c|c|}
\hline PIEZA RECLAMAADA & USADA CONO & MATERIA PRIMA & TAMANO & MODULO \\
\hline $\begin{array}{l}\text { Núcleo piramidal } \\
\text { irregular }\end{array}$ & $\begin{array}{l}\text { Sin cambio en la } \\
\text { función }\end{array}$ & Andesita G & Grandisimo ${ }^{*}$ & $\begin{array}{l}\text { Mediano } \\
\text { alargado }\end{array}$ \\
\hline Núcleo bifacial & $\begin{array}{l}\text { Sin cambio en la } \\
\text { función }\end{array}$ & Andesita G & Grandisimo & $\begin{array}{l}\text { Mediano } \\
\text { alargado }\end{array}$ \\
\hline Núcleo bifacial & $\begin{array}{l}\text { Sin cambio en } \\
\text { función }\end{array}$ & Andesita G & Grandisimo & Corto ancho \\
\hline $\begin{array}{l}\text { Con lascados } \\
\text { aislados }\end{array}$ & $\begin{array}{l}\text { Sin cambio en la } \\
\text { función }\end{array}$ & Andesita B & Muy grande & Corto anchisimo \\
\hline Discoidal irregular & $\begin{array}{l}\text { Sin cambio en la } \\
\text { función }\end{array}$ & Andesita G & Grande & Corto muy ancho \\
\hline $\begin{array}{c}\text { Núcleo prismático } \\
\text { parcial }\end{array}$ & $\begin{array}{l}\text { Sin cambio en la } \\
\text { función }\end{array}$ & Andesita B & Muy grande & $\begin{array}{l}\text { Mediano } \\
\text { alargado }\end{array}$ \\
\hline $\begin{array}{l}\text { Fragmento no } \\
\text { diferenciado de } \\
\text { núcleo }\end{array}$ & $\begin{array}{l}\text { Sin cambio en la } \\
\text { función }\end{array}$ & Andesita B & Muy grande & Corto ancho \\
\hline $\begin{array}{l}\text { Fragmento no } \\
\text { diferenciado de } \\
\text { núcleo }\end{array}$ & $\begin{array}{l}\text { Sin cambio en la } \\
\text { función }\end{array}$ & Andesita G & $\begin{array}{l}\text { Mediano } \\
\text { pequefto }\end{array}$ & Mediano normal \\
\hline
\end{tabular}

Tabla 3. Características generales de los núcleos con evidencias de reclamación.

Estos conjuntos fueron tallados in situ, y su depositación cultural refleja en cierto sentido, una situación de 'abandono' o 'descarte'. Estos conjuntos de artefactos constituyen áreastalleres que, a su vez, conforman una cantera para el aprovisionamiento, asociados a la fuente secundaria que los contiene. Los conjuntos líticos de estas áreas-taller se suman a la oferta lítica de la fuente secundaria en sí misma, convirtiéndose estos conjuntos en una nueva fuente de aprovisionamiento (contenida en la secundaria), es decir en una suerte de fuente terciaria (Church 1995).

Esto hace que dentro de un mismo espacio estén operando dos tipos de fuentes -secundaria y terciaria-, donde la materia prima se encuentra disponible bajo formas bastante diferentes: clastos naturales y artefactos líticos. La evidencia de utilización posterior al 'abandono' o 'descarte' de ciertos artefactos en la fuente secundaria (reclamación) es la que obliteraría la 


\begin{tabular}{|c|c|c|c|c|}
\hline Pieza Reclamada & $\begin{array}{l}\text { USADA PARA CONFECCION } \\
\text { DE }\end{array}$ & $\begin{array}{l}\text { MATERIA } \\
\text { PRIMA }\end{array}$ & TAMANO & Mooulo \\
\hline Lasca entera: & Muesca de lastado simplo & Andesifa G & Grande: & $\begin{array}{l}\text { Corto muy } \\
\text { ancho }\end{array}$ \\
\hline Lasca entera & Muesca de lascado simple & Andesita B & $\begin{array}{l}\text { Muy } \\
\text { grande }\end{array}$ & $\begin{array}{l}\text { Corto muy } \\
\text { anche }\end{array}$ \\
\hline Lasca entera & Muesca telocads & Andesita B & $\begin{array}{l}\text { Muy } \\
\text { grande }\end{array}$ & Mediane nctmal \\
\hline Lasca entera & Chopper & Andesita $B$ & Grandigimo & $\begin{array}{l}\text { Mediano } \\
\text { alargado }\end{array}$ \\
\hline $\begin{array}{c}\text { Filo bfacial de anista } \\
\text { simuosa }\end{array}$ & $\begin{array}{l}\text { Rasefera + Muesca de } \\
\text { lascado simple }\end{array}$ & Andesta B & $\begin{array}{l}\text { Mury } \\
\text { grande }\end{array}$ & $\begin{array}{l}\text { Mediano } \\
\text { alargado }\end{array}$ \\
\hline Biface parcial & $\begin{array}{l}\text { Raedera + Muesca refocada + } \\
\text { muesca de lascado simple }\end{array}$ & Andesita 0 & $\begin{array}{l}\text { May } \\
\text { grande }\end{array}$ & $\begin{array}{l}\text { Mediano } \\
\text { alargado }\end{array}$ \\
\hline Biface parcial & Muesca relocada & Andesita B & Grandisimo & $\begin{array}{l}\text { Mediano } \\
\text { alargado }\end{array}$ \\
\hline Biface parcial & Muesca de lascado simple & Andesita $G$ & Grandisimo: & Laminar nomal \\
\hline Biface parcial & Muesca rebocada & Andesida G & $\begin{array}{l}\text { Muy } \\
\text { grande }\end{array}$ & Mediano normal \\
\hline Biface parcial & $\begin{array}{c}\text { Denticulado + Punta entre } \\
\text { muescas }\end{array}$ & Andesita G & $\begin{array}{l}\text { May } \\
\text { grande }\end{array}$ & $\begin{array}{l}\text { Mediano } \\
\text { alargado }\end{array}$ \\
\hline Esbozo de pieza bifacial & $\begin{array}{l}\text { Chopper + Muesca de } \\
\text { lascado simple }\end{array}$ & Andesita $B$ & Crandisimo & Mediano normal \\
\hline Estozo de pieza bifacial & Deniticulado & Andesita G & Grandismo & $\begin{array}{l}\text { Mediano } \\
\text { alargado }\end{array}$ \\
\hline Biface sensu stricto. & Denticulado. & Andesta 0 & Grandiamo & $\begin{array}{l}\text { Mediano } \\
\text { alargado }\end{array}$ \\
\hline
\end{tabular}

Tabla 4. Características generales de los artefactos formatizados con evidencias de reclamación.

noción de fuente secundaria en un sentido estricto, siendo el concepto de fuente terciaria más adecuado para describir situaciones como la planteada. Esta situación no impide reconocer que ambos tipos de fuentes pudieron funcionar en el mismo espacio (superficie glacis) y, en algún momento, de forma simultánea.

En relación al aspecto temporal del uso de esta fuente, ciertos artefactos del sitio fueron datados mediante la técnica VML que establece edades mínimas de exposición de las superficies sobre las que yacen los conjuntos líticos. Los resultados de las dataciones obtenidas sobre piezas líticas del sitio Planchada La Puntilla, indican que la capa más antigua del barniz se depositó hace, al menos, 5900 años AP (Somonte 2009). Esto estaría indicando una antigüedad mayor de los artefactos, aunque no se pueda precisar la cronología exacta de producción ni de reclamación de los mismos.

La superficie del glacis donde se encuentra emplazado el sitio Planchada La Puntilla conforma una fuente secundaria que, en algún momento anterior a la construcción de los recintos, fue utilizada como lugar de aprovisionamiento y donde algunos sectores fueron 
convertidos en taller. Lo anterior, sumado a la ausencia de evidencias de reclamación en el interior del recinto R4 y la ausencia de materiales arqueológicos en los restantes recintos excavados, en conjunto invita a postular que la dinámica ocupacional de este sitio estuvo dada fundamentalmente en relación al uso de la fuente en sí misma y el aprovisionamiento de recursos en estado natural así como otros bajo la forma de artefactos dejados en la fuente. Además, en base al fechado de 5900 años AP, es probable que esta explotación haya tenido lugar con bastante anterioridad a la construcción y 'ocupación' de los recintos en sí mismos, dada la no-contemporaneidad entre el uso inicial del espacio fuente y la construcción y 'ocupación' de los recintos.

En este sentido, las evidencias de reclamación en Planchada La Puntilla, observadas en piezas afectadas por barniz de las rocas con diversas intensidades, advierten acerca de las numerosas visitas que ha tenido este espacio a lo largo del tiempo y donde las reclamaciones están asociadas a la explotación de los recursos líticos allí presentes y no tanto al ingreso desde otras localizaciones de piezas retomadas.

\section{A modo de cierre}

Entender el espacio donde se emplaza el sitio arqueológico Planchada La Puntilla es crucial para abordar el estudio de la historia ocupacional de numerosos sitios de Amaicha del Valle. Es recurrente en el área la presencia de sitios que poseen la particularidad de conformar el lugar de emplazamiento de diversos asentamientos prehispánicos, cuyos ocupantes se proveyeron de materia prima en los alrededores inmediatos de sus viviendas y bajo diversas formas.

Esto da lugar a la presencia en estos sitios, de los cuales Planchada La Puntilla es sólo un ejemplo, de cuatro situaciones a considerar: (1) fuente de aprovisionamiento; (2) áreas de explotación de recursos líticos (una especie de áreas 'cantera-taller') correspondientes a la fuente secundaria; ( 3 ) sectores dentro de estas áreas de talleres utilizadas como fuente terciaria; y (4) sector de recintos que no responderían a típicas bases residenciales (Somonte 2009). Estas cuatro instancias están asociadas a restos arqueológicos cuyas características remiten a situaciones que aluden a distintos momentos en el uso/explotación y ocupación de ese espacio. Esto es interpretado como una superposición en el espacio de temporalidades claramente diferentes, marcada por la evidencia de explotación de la fuente por parte de gentes distintas.

\section{Agradecimientos}

Este trabajo es resultado de la investigación realizada en el marco de los Proyectos CIUNT G-328 y G-406, financiados por la Secretaria de Ciencia y Técnica de la Universidad Nacional de Tucumán. Nuestro reconocimiento al señor Marcos Pastrana, por permitirnos trabajar en el sitio Planchada La Puntilla y por su hospitalidad y apoyo a nuestro trabajo; a la Comunidad Indígena Amaicha del Valle, por acompañar nuestro trabajo; a los evaluadores, por sus sugerencias que ayudaron a mejorar el manuscrito. Lo vertido en el texto, no obstante, es exclusiva responsabilidad de los autores. 
Notas

${ }^{1}$ El Complejo Portezuelo de las Ánimas corresponde a los afloramientos de rocas volcánicas ubicados entre los 3600 y $4384 \mathrm{msnm}$. Se encuentra en la parte más alta de la sierra del Aconquija -en el flanco occidental- y está constituido por una secuencia alternante de brechas piroclásticas y lapillíticas de composición andesítica. Las brechas ocupan una extensión de $50 \mathrm{~km} 2$ y poseen tamaños de clastos pequeños hasta grandes bloques; siendo estos últimos los más frecuentes (González 1990).

${ }^{2}$ Con respecto a la presencia de las andesitas en el área del valle de Santa María y Amaicha, en general, existe un consenso (en base a lo que se lee en las tesis y publicaciones de miembros de los equipos de investigación de Myriam Tarragó por un lado, Cristina Scattolin por otro y, recientemente, Nurit Oliszewski), en que las rocas volcánicas de carácter local, presentes en los conjuntos líticos de diversos sitios, son en términos generales andesitas (Lazzari 2006; Funes Coronel 2007; Carbonelli 2009). Particularmente, se debe aclarar que Marisa Lazzari (2006) ha detectado mediante análisis químicos una andesita que también denominó andesita B (relacionada a las 'piezas basálticas' de la Ciénaga), que no se sabe aún si corresponde o no a la denominada por nosotros de la misma manera. En este sentido, la similitud en las designaciones solamente es mera coincidencia y no debe interpretarse esta información en referencia a un mismo tipo de recurso lítico. Sobre este aspecto se trabajará en un futuro cercano.

\section{Bibliografía citada}

Aschero, C.A

1975. Ensayo para una clasificación morfológica de artefactos líticos aplicada a estudios tipológicos interpretativos. Informe inédito presentado al CONICET. Manuscrito.

1983. Ensayo para una clasificación morfológica de artefactos líticos. Apéndices A y B. Apunte inédito para la cátedra de Ergología y Tecnología. Facultad de Filosofía y Letras. Universidad de Buenos Aires. Manuscrito.

Aschero, C.A. y S. Hocsman

2004. Revisando cuestiones tipológicas en torno a la clasificación de artefactos bifaciales. Temas de Arqueología. Análisis lítico. (comp. por M. Ramos, A. Acosta y D. Loponte), pp. 7-25. Universidad Nacional de Luján. Luján.

Borrazzo, K.

2006. Tafonomía lítica en dunas: una propuesta para el análisis de los artefactos líticos. Intersecciones en Antropología 7: 247-261

Bossi, G.E., A. Villlanueva García, M.H. Carrión, R.M. Palma y J.I. Díaz 1984. El grupo Santa María en la Quebrada de Amaicha (Depto. Tafí, Pcia. Tucumán). Actas del Noveno Congreso Geológico Argentino, Tomo I, pp. 124-241. Bariloche.

Bossi, G., I. Gavriloff y G. Esteban 1998. Terciario (Estratigrafía, Bioestratigrafía y Paleogeografía). Geología de Tucumán (ed. por M. Gianfrancisco, M.E. Puchulu, J. Durango de Cabrera y G.F. Aceñolaza), pp. 87-110. $2^{\circ}$ edición. Colegio de Graduados en Ciencias Geológicas, Tucumán. 
Carbonelli, J.P.

2009. Interacciones cotidianas entre materias primas y sujetos sociales en el Valle de Yocavil. El caso del sitio Soria 2 (Andalhuala, Pcia. de Catamarca). Tesis de Licenciatura en Ciencias Antropológicas, Facultad de Filosofía y Letras. Universidad de Buenos Aires.

Civalero, M.T. y N.V. Franco

2003. Early human occupations in western Santa Cruz Province, southernmost South America. Quaternary International 109-110: 77-86.

Church, T.

1995. Terms in lithic resource studies. Lithic resource studies: a source for archaeologist. Special Publication, Department of Antrhopology. Lithic Technology 3: 9-25. University of Tulsa, Oklahoma.

Dorn, R.

2007. Rock Varnish. Geochemical Sediments and Landscapes (ed. por D. J. Nash y S.J. McLaren), pp. 246-297. Blackwell, London.

Funes Coronel, J.A.

2007. Caracterización del conjunto lítico del sitio Cueva de Los Corrales 1 (CC1), El Infiernillo, Tucumán. Primeras Jornadas de Jóvenes Investigadores UNT-AUGM. Tucumán. Consejo de Investigaciones de la Universidad Nacional de Tucumán.

Gavriloff, I.; J. Durango de Cabrera y M. Vergel

1998. Paleontología de invertebrados, paleobotánica y palinología. Geología de Tucumán (ed. por M. Gianfrancisco, M.E. Puchulu, J. Durango de Cabrera y G. Aceñolaza), pp. 211-226. $2^{\circ}$ Edición. Colegio de Graduados en Ciencias Geológicas de Tucumán, Tucumán.

González, O.E.

1990. Las volcanitas del Portezuelo de las Ánimas, Sierra de Aconquija, Provincias de Catamarca y Tucumán. Revista de la Asociación Geológica Argentina XLV (3-4):386-396.

González, O.E.; M.E. Viruel; R. Mon y P. Tchilinguirian

2000. Hoja Geológica 2766-II San Miguel de Tucumán. Provincias de Tucumán, Catamarca, Salta y Santiago del Estero. E: 1:250.000. Programa Nacional de Cartas Geológicas de la República Argentina.

González, O.E. y O. Barreñada

1993. Geología y Estructura de las Nacientes del río Amaicha y el Infiernillo, Provincia de Tucumán. En Actas XII Congreso Geológico Argentino y II Congreso de Exploración de Hidrocarburos, Tomo III, pp. 72-81. Mendoza.

Hiscock, P.

2007. Looking the other way. A materialist/technological approach to classifying tools and implements cores and retouched flakes. Tools versus Cores?Alternative approaches to Stone Tool Analysis (ed. por S. McPherron), pp. 198-222. Cambridge Scholars Publishing, Cambridge. 
Lazzari, M.

2006. Travelling things and the production of social spaces: An archaeological study of circulation and value in North Western Argentina. Ph.D. Dissertation. Graduate School of Arts and Sciences, Columbia University.

Liu, T.

2003. Blind testing of rock varnish microstratigraphy as a chronometric indicator: Results on late Quaternary lava flows in the Mojave Desert, California. Geomorphology 53: 209-234.

Liu, T. y W.S. Broecker

2008. Rock varnish microlamination dating of late Quaternary geomorphic features in the drylands of western USA. Geomorphology 93: 501-523.

2007. Holocene rock varnish microstratigraphy and its chronometric application in the drylands of western USA. Geomorphology 84: 1-21.

2000. How fast does rock varnish grow? Geology 28: 183-186.

Liu, T. y. R. Dorn

1996. Understanding spatial variability in environmental changes in drylands with rock varnish microlaminations. Annals of the Association of American Geographers 86: 187-212.

Luedtke, B.

1979. The identification of sources of chert artifacts. American Antiquity 44: 744-756.

Nami, H.G.

1992. El subsistema tecnológico de la confección de instrumentos líticos y la explotación de los recursos del ambiente: Una nueva vía de aproximación. Shincal 2: 33-53.

Schiffer, M.

1987. Formation Processes of the Archaeological Record. University of New Mexico Press, Albuquerque.

Somonte, $\mathrm{C}$.

2009. Tecnología lítica en espacios persistentes de Amaicha del Valle (Tucumán). Tesis Doctoral. Facultad de Filosofia y Letras, Universidad de Buenos Aires.

Somonte C. y M. Collantes

2007. Barniz de las rocas y espacios persistentes: su abordaje desde los procesos de reclamación artefactual lítica en Amaicha del Valle (Tucumán). Mundo de Antes 5: 119-137. 
